doi:10.13108/2021-13-2-22

\title{
INTEGRALS AND CHARACTERISTIC LIE RINGS OF SEMI-DISCRETE SYSTEMS OF EQUATIONS
}

\author{
A.V. ZHIBER, M.N. KUZNETSOVA
}

\begin{abstract}
The paper is devoted to studying systems of semi-discrete equations $\bar{r}_{n+1, x}=$ $\bar{h}\left(x, n, \bar{r}_{n}, \bar{r}_{n+1}, \bar{r}_{n, x}\right)$ within the framework of an approach based on the concept of a characteristic Lie ring. Here $\bar{r}_{n}=\left(r_{n}^{1}, r_{n}^{2}, \ldots, r_{n}^{N}\right), \bar{h}=\left(h^{1}, h^{2}, \ldots, h^{N}\right), n \in \mathbb{Z}$. Among integrable nonlinear partial differential equations and systems, we find Darboux integrable nonlinear hyperbolic equations and systems. A feature of such equations is the existence of integrals along each characteristic direction, the so-called $x$ - and $y$-integrals. This allows us to reduce the integration of a partial differential equation to integrating a system of ordinary differential equations. Darboux integrable equations and systems can be efficiently studied and classified by means of characteristic Lie rings. Papers by Leznov, Smirnov, Shabat, Yamilov underlie an algebraic approach for studying nonlinear hyperbolic systems. Currently, the algebraic approach is extended to semi-discrete and discrete equations. In this paper, we prove that the system has $N$ essentially independent $x$-integrals if and only if the characteristic Lie ring corresponding to a continuous characteristic direction is finitedimensional.
\end{abstract}

Keywords:semi-discrete system of equations, characteristic ring, $x$-integral, Darboux integrable system.

Mathematics Subject Classification: 37K10, 37K30, 37D99

\section{INTRODUCTION}

This work is devoted to studying systems of semi-discrete equations

$$
\bar{r}_{n+1, x}=\bar{h}\left(x, n, \bar{r}_{n}, \bar{r}_{n+1}, \bar{r}_{n, x}\right)
$$

in the framework of an approach based on the notion of the characteristic Lie ring. Here $\bar{r}_{n}=\left(r_{n}^{1}, r_{n}^{2}, \ldots, r_{n}^{N}\right), \bar{h}=\left(h^{1}, h^{2}, \ldots, h^{N}\right), n \in \mathbb{Z}$.

We first of all give rigorous definitions and formulations of the results. We begin with independent variables. Each identity is to hold identically on each solution of system (1.1). This is why everywhere we replace $\bar{r}_{n+1, x}$ by the right hand side $\bar{h}\left(x, n, \bar{r}_{n}, \bar{r}_{n+1}, \bar{r}_{n, x}\right)$, and $\bar{r}_{n+2, x}$ is replaced by $\bar{h}\left(x, n+1, \bar{r}_{n+1}, \bar{r}_{n+2}, \bar{h}\left(x, n, \bar{r}_{n}, \bar{r}_{n+1}, \bar{r}_{n, x}\right)\right)$ and so forth. Thus, the independent variables are

$$
\ldots, r_{n-m}^{i}, \ldots, r_{n-1}^{i}, r_{n}^{i}, r_{n+1}^{i}, \ldots, r_{n+k}^{i}, \ldots, r_{n, x}^{i}, r_{n, x x}^{i}, r_{n, x x x}^{i}, \ldots
$$

Hereafter we employ the notation $D_{x}$ for the operator of total differentiating in the variable $x$ and $D$ denotes the operator of the shift by one over $n$, that is,

$$
\begin{array}{ll}
\operatorname{Dr}(n, x)=r(n+1, x), & D^{-1} r(n, x)=r(n-1, x), \\
D^{2} r(n, x)=r(n+2, x), & D^{-2} r(n, x)=r(n-2, x) .
\end{array}
$$

A.V. Zhiber, M.N. KuZnetsova, Integrals And CHARACTERISTiC Lie Rings Of SEMI-DisCRETE SYSTEMS OF EQUATIONS.

(C) Zhiber A.V., Kuznetsova M.N. 2021.

The research is supported by a grant of Russian Science Foundation (project no. 21-11-00006).

Submitted April 15, 2021. 
The derivatives of the vector $\bar{r}_{n}$ are denoted by $\bar{r}_{n, x}=\left(r_{n, x}^{1}, r_{n, x}^{2}, \ldots, r_{n, x}^{N}\right), \ldots$,

$$
\bar{r}_{n}^{(m)}=\left(r_{n}^{1,(m)}, \ldots, r_{n}^{N,(m)}\right)=\left(\frac{\partial^{m} r_{n}^{1}}{\partial x^{m}}, \ldots, \frac{\partial^{m} r_{n}^{N}}{\partial x^{m}}\right)
$$

Definition 1.1. A function

$$
W=W\left(x, n, \bar{r}_{n}, \bar{r}_{n+1}, \ldots, \bar{r}_{n+s}\right), \quad \sum_{i=1}^{N}\left(\frac{\partial W}{\partial r_{n+s}^{i}}\right)^{2} \neq 0
$$

obeying a characteristic equation $D_{x} W=0$ is called $x$-integral of system (1.1) and the numbers $s$ is its order.

Definition 1.2. A function

$$
I=I\left(x, n, \bar{r}_{n}, \bar{r}_{n, x}, \bar{r}_{n, x x}, \ldots, \bar{r}_{n}^{(m)}\right), \quad \sum_{i=1}^{N}\left(\frac{\partial I}{\partial r_{n}^{i,(m)}}\right)^{2} \neq 0
$$

obeying equation $D I=I$ is called n-integral of system (1.1) and the number $m$ is its order.

System of equations (1.1) is called Darboux integrable if possesses a complete set of functionally independent integrals, $N$ integrals in each characteristic direction.

Example 1.1. A chain

$$
t_{n+1, x}=t_{n, x}+c e^{\frac{t_{n}+t_{n+1}}{2}}
$$

is Darboux integrable since it admits an $x$-integral

$$
W=e^{\frac{t_{n+1}-t_{n}}{2}}+e^{\frac{t_{n+1}-t_{n+2}}{2}}
$$

and an n-integral (see [3])

$$
I=t_{n, x x}-\frac{t_{n, x}^{2}}{2}
$$

Darboux integrable equations and systems can be effectively studied and classified by means of characteristic Lie rings.

The notion of a characteristic Lie algebra was introduced in work [1] for systems of hyperbolic equations of form

$$
u_{x y}^{i}=F^{i}(u), \quad i=1,2, \ldots, n .
$$

In works [1], 2], a criterion for the Darboux integrability was proved for nonlinear hyperbolic systems of equations. It was shown that system (1.3) possesses a complete set of integrals if and only if its characteristic algebra is finite dimensional. In work [4], a criterion of the Darboux integrability was obtained for nonlinear hyperbolic systems of equations of form

$$
u_{x y}=F\left(u, u_{x}, u_{y}\right) \quad\left(u_{x y}^{i}=F^{i}, i=1,2, \ldots, n\right) .
$$

In works [5], 6], a notion of a characteristic ring of a discrete equation was introduced and by means of this notions, Darboux integrable differential-difference equations of form $u_{i+1, x}=$ $f\left(u_{i}, u_{i+1}, u_{i, x}\right)$ were classified. Works [7], [8] were devoted to constructing a complete set of integrals for a hyperbolic system. In work [9] a conjecture was formulated: a system of equations (1.1) possesses a complete set of $x$ - and $n$-integrals if and only if the characteristic ring in each characteristic direction is finite-dimensional.

In the present work we prove that system (1.1) possesses $N$ independent $x$-integrals if and only if the characteristic ring in this characteristic direction is finite-dimensional.

The paper is organized as follows. Section 2 provides the proof of the main result formulated in Theorem 2.1 for system (1.1) as $N=2$. In Section 3 we give a scheme of the proof of the main result for an arbitrary $N$ (Theorem 3.1). In Conclusion we discuss the results. 


\section{Characteristic Rings. Case $N=2$}

Here we study the case $N=2$ :

$$
\bar{r}_{n+1, x}=\bar{h}\left(x, n, \bar{r}_{n}, \bar{r}_{n+1}, \bar{r}_{n, x}\right), \quad \bar{r}_{n}=\left(r_{n}^{1}, r_{n}^{2}\right), \quad \bar{h}=\left(h^{1}, h^{2}\right), \quad n \in \mathbb{Z} .
$$

Let us find Lie $x$-ring of system (2.1). On the set of locally-analytic functions depending on the variables $x, \bar{r}_{n, x}, \bar{r}_{n}, \bar{r}_{n+1}, \ldots$ the operator of total differentiation in $x$ reads as

$$
D_{x}=\frac{\partial}{\partial x}+r_{n, x x}^{1} \frac{\partial}{\partial r_{n, x}^{1}}+r_{n, x x}^{2} \frac{\partial}{\partial r_{n, x}^{2}}+\sum_{k=0}^{\infty}\left(r_{n+k, x}^{1} \frac{\partial}{\partial r_{n+k}^{1}}+r_{n+k, x}^{2} \frac{\partial}{\partial r_{n+k}^{2}}\right) .
$$

By system of equations 2.1) we obtain the relations

$$
\bar{r}_{n+k, x}=\bar{h}_{n+k}\left(x, n, \bar{r}_{n}, \bar{r}_{n+1}, \ldots, \bar{r}_{n+k}, \bar{r}_{n, x}\right), \quad k=1,2, \ldots
$$

We represent operator 2.2 as

$$
D_{x}=r_{n, x x}^{1} Y_{1}+r_{n, x x}^{2} Y_{2}+Y_{3},
$$

where

$$
Y_{1}=\frac{\partial}{\partial r_{n, x}^{1}}, \quad Y_{2}=\frac{\partial}{\partial r_{n, x}^{2}}, \quad Y_{3}=\frac{\partial}{\partial x}+\sum_{k=0}^{\infty}\left(r_{n+k, x}^{1} \frac{\partial}{\partial r_{n+k}^{1}}+r_{n+k, x}^{2} \frac{\partial}{\partial r_{n+k}^{2}}\right) .
$$

According formulae (2.3), the vector field $Y_{3}$ can be represented as

$$
Y_{3}=\frac{\partial}{\partial x}+r_{n, x}^{1} \frac{\partial}{\partial r_{n}^{1}}+r_{n, x}^{2} \frac{\partial}{\partial r_{n}^{2}}+\sum_{k=1}^{\infty}\left(\alpha_{k} \frac{\partial}{\partial r_{n+k}^{1}}+\beta_{k} \frac{\partial}{\partial r_{n+k}^{2}}\right),
$$

where $\bar{h}_{n+k}=\left(\alpha_{k}, \beta_{k}\right)$. We note that

$$
\alpha_{k}=\alpha_{k}\left(x, n, \bar{r}_{n}, \bar{r}_{n+1}, \ldots, \bar{r}_{n+k}, \bar{r}_{n, x}\right), \quad \beta_{k}=\beta_{k}\left(x, n, \bar{r}_{n}, \bar{r}_{n+1}, \ldots, \bar{r}_{n+k}, \bar{r}_{n, x}\right) .
$$

According (2.4), the characteristic equation

$$
D W\left(x, n, \bar{r}_{n}, \bar{r}_{n+1}, \ldots, \bar{r}_{n+m}\right)=0
$$

is equivalent to system

$$
Y_{1} W=0, \quad Y_{2} W=0, \quad Y_{3} W=0 .
$$

With equations (2.6), a Lie ring generated by the vectors fields $Y_{1}, Y_{2}$ and $Y_{3}$ is naturally associated. We shall call this ring $\mathcal{X}$ a characteristic Lie $x$-ring of system of equations (2.1). Solutions of equations (2.5) will be called $x$-integrals.

The following statement holds.

Lemma 2.1. If system of equations 2.1) possesses two essentially independent $x$-integrals, then the ring $\mathcal{X}$ is finite-dimensional.

Proof. Assume that system 2.1) possesses a pair of essentially independent integrals of the same order

$$
\omega\left(x, n, \bar{r}_{n}, \ldots, \bar{r}_{n+m}\right), \quad W\left(x, n, \bar{r}_{n}, \ldots, \bar{r}_{n+m}\right),
$$

that is,

Then the identity holds:

$$
\left|\begin{array}{cc}
\frac{\partial \omega}{\partial r_{n+m}^{1}} & \frac{\partial \omega}{\partial r_{n+m}^{2}} \\
\frac{\partial W}{\partial r_{n+m}^{1}} & \frac{\partial W}{\partial r_{n+m}^{2}}
\end{array}\right| \neq 0
$$

$$
\bar{r}_{n+m}=\bar{a}_{n}\left(\omega, W, x, n, \bar{r}_{n}, \ldots, \bar{r}_{n+m-1}\right) .
$$


We let $\omega_{n}=\omega, W_{n}=W$. Then by (2.7) we obtain the relations

$$
\bar{r}_{n+m+k}=\bar{A}_{k}\left(x, n, \omega_{n}, W_{n}, \ldots, \omega_{n+k}, W_{n+k}, \bar{r}_{n}, \bar{r}_{n+1}, \ldots, \bar{r}_{n+m-1}\right), \quad k=0,1,2, \ldots
$$

Thus, taking into consideration formulae (2.8), we can pass from independent variables

$$
\bar{r}_{n, x}, x, \bar{r}_{n}, \bar{r}_{n+1}, \ldots, \bar{r}_{n+s}, \ldots
$$

to new variables

$$
\bar{r}_{n, x}, x, \bar{r}_{n}, \bar{r}_{n+1}, \ldots, \bar{r}_{n+m-1}, \omega_{n}, W_{n}, \ldots, \omega_{n+k}, W_{n+k}, \ldots
$$

In new variables (2.10), the operator $Y_{3}$ is written as

$$
\tilde{Y}_{3}=\frac{\partial}{\partial x}+\sum_{k=0}^{m-1}\left(r_{n+k, x}^{1} \frac{\partial}{\partial r_{n+k}^{1}}+r_{n+k, x}^{2} \frac{\partial}{\partial r_{n+k}^{2}}\right) \text {. }
$$

Under the change of variables, the relation $\overline{[X, Z]}=[\bar{X}, \bar{Z}]$ holds, where the bar means an initial operator in new variables, and the Lie ring generated by the operators $Y_{1}, Y_{2}$ and $\tilde{Y}_{3}$ is finite-dimensional. This is the initial Lie $x$-ring $\mathcal{X}$ is finite-dimensional.

Let initial system (2.1) possesses a pair of essentially independent integrals of different minimal order

$$
\omega\left(x, n, \bar{r}_{n}, \ldots, \bar{r}_{n+l}\right), \quad W\left(x, n, \bar{r}_{n}, \ldots, \bar{r}_{n+m}\right), \quad l<m .
$$

The latter means that the integrals

$$
\omega\left(x, n+m-l, \bar{r}_{n+m-l}, \ldots, \bar{r}_{n+m}\right), \quad W\left(x, n, \bar{r}_{n}, \ldots, \bar{r}_{n+m}\right)
$$

are essentially independent. Passing then from variables $(2.9)$ to the variables

$$
\bar{r}_{n, x}, x, \bar{r}_{n}, \bar{r}_{n+1}, \ldots, \bar{r}_{n+m-1}, \omega_{n+m-l}, \omega_{n+m-l+1}, \ldots, W_{n}, W_{n+1}, \ldots,
$$

as above, we obtain that the ring $\mathcal{X}$ is finite dimensional. The proof is complete.

Let us consider the issue on essentially independence of two integrals. Suppose that integrals 2.11) are essentially dependent. This means that the identity holds:

$$
W\left(x, n, \bar{r}_{m}, \ldots, \bar{r}_{n+m}\right)=F\left(x, n, \bar{r}_{n}, \ldots, \bar{r}_{n+m-1}, \omega\left(x, n+m-l, \bar{r}_{n+m-l}, \ldots, \bar{r}_{n+m}\right)\right) .
$$

Hence,

$$
W=\sum_{k=0}^{\infty} F_{k}\left(x, n, \bar{r}_{n}, \ldots, \bar{r}_{n+m-1}\right)\left(\omega-\omega^{0}\right)^{k} .
$$

Since $\omega$ and $W$ are integrals of minimal order we obtain that

$$
F_{k}=\Phi_{k}\left(x, n, \omega\left(x, n, \bar{r}_{n}, \ldots, \bar{r}_{n+l}\right), \ldots, \omega\left(x, n+m-l, \bar{r}_{n+m-l}, \ldots, \bar{r}_{n+m-1}\right)\right) .
$$

Thus,

$$
W=\Phi\left(x, n, \omega_{n}, \omega_{n+1}, \ldots, \omega_{n+m-l}\right)
$$

and initial system (1.1) possesses just one $x$-integrals.

We proceed to inverse problem. Let the ring $\mathcal{X}$ be finite-dimensional. It is clear that $\operatorname{dim} \mathcal{X} \geqslant$ 5 .

We consider the case $\operatorname{dim} \mathcal{X}=5$. Then the basis of the ring $\mathcal{X}$ is defined by the vector fields $Y_{1}, Y_{2}, Y_{3}, Y_{13}=\left[Y_{1}, Y_{3}\right], Y_{23}=\left[Y_{2}, Y_{3}\right]$. Since

$$
\begin{aligned}
Y_{3}= & \frac{\partial}{\partial x}+r_{n, x}^{1} \frac{\partial}{\partial r_{n}^{1}}+r_{n, x}^{2} \frac{\partial}{\partial r_{n}^{2}} \\
& +\sum_{k=1}^{\infty}\left(\alpha_{k}\left(x, n, \bar{r}_{n}, \bar{r}_{n+1}, \ldots, \bar{r}_{n+k}, \bar{r}_{n, x}\right) \frac{\partial}{\partial r_{n+k}^{1}}\right. \\
& \left.+\beta_{k}\left(x, n, \bar{r}_{n}, \bar{r}_{n+1}, \ldots, \bar{r}_{n+k}, \bar{r}_{n, x}\right) \frac{\partial}{\partial r_{n+k}^{2}}\right)
\end{aligned}
$$


we have

$$
\begin{aligned}
& {\left[Y_{1}, Y_{3}\right]=\frac{\partial}{\partial r_{n}^{1}}+\sum_{k=1}^{\infty}\left(\frac{\partial \alpha_{k}}{\partial r_{n, x}^{1}} \frac{\partial}{\partial r_{n+k}^{1}}+\frac{\partial \beta_{k}}{\partial r_{n, x}^{1}} \frac{\partial}{\partial r_{n+k}^{2}}\right)} \\
& {\left[Y_{2}, Y_{3}\right]=\frac{\partial}{\partial r_{n}^{2}}+\sum_{k=1}^{\infty}\left(\frac{\partial \alpha_{k}}{\partial r_{n, x}^{2}} \frac{\partial}{\partial r_{n+k}^{1}}+\frac{\partial \beta_{k}}{\partial r_{n, x}^{2}} \frac{\partial}{\partial r_{n+k}^{2}}\right)}
\end{aligned}
$$

Then we replace vector field $(2.12)$ by

$$
\tilde{Y}_{3}=Y_{3}-r_{n, x}^{1} Y_{13}-r_{n, x}^{2} Y_{23}
$$

Thus, we have the following bases:

$$
\begin{aligned}
& Y_{1}=\frac{\partial}{\partial r_{n, x}^{1}}, \quad Y_{2}=\frac{\partial}{\partial r_{n, x}^{2}}, \quad \tilde{Y}_{3}=\frac{\partial}{\partial x}+\sum_{k=1}^{\infty}\left(\tilde{\alpha}_{k} \frac{\partial}{\partial r_{n+k}^{1}}+\tilde{\beta}_{k} \frac{\partial}{\partial r_{n+k}^{2}}\right), \\
& Y_{13}=\frac{\partial}{\partial r_{n}^{1}}+\sum_{k=1}^{\infty}\left(\gamma_{k} \frac{\partial}{\partial r_{n+k}^{1}}+\delta_{k} \frac{\partial}{\partial r_{n+k}^{2}}\right), \quad Y_{23}=\frac{\partial}{\partial r_{n}^{2}}+\sum_{k=1}^{\infty}\left(p_{k} \frac{\partial}{\partial r_{n+k}^{1}}+q_{k} \frac{\partial}{\partial r_{n+k}^{2}}\right) .
\end{aligned}
$$

It is easy to see that the coefficients $\tilde{\alpha}_{k}, \tilde{\beta}_{k}, \gamma_{k}, \delta_{k}, p_{k}, q_{k}$ are independent of the variables $r_{n, x}^{1}$ and $r_{n, x}^{2}$ and they are the functions on the variables $x, n, \bar{r}_{n}, \ldots, \bar{r}_{n+k}$ otherwise $\operatorname{dim} \mathcal{X}>5$. According (2.13), characteristic equation (2.5) for $x$-integral $W\left(x, n, \bar{r}_{n}, \bar{r}_{n+1}\right)$ is reduced to system of equations

$$
\begin{aligned}
& \left(\frac{\partial}{\partial x}+\tilde{\alpha}_{1} \frac{\partial}{\partial r_{n+1}^{1}}+\tilde{\beta}_{1} \frac{\partial}{\partial r_{n+1}^{2}}\right) W=0 \\
& \left(\frac{\partial}{\partial r_{n}^{1}}+\gamma_{1} \frac{\partial}{\partial r_{n+1}^{1}}+\delta_{1} \frac{\partial}{\partial r_{n+1}^{2}}\right) W=0 \\
& \left(\frac{\partial}{\partial r_{n}^{2}}+p_{1} \frac{\partial}{\partial r_{n+1}^{1}}+q_{1} \frac{\partial}{\partial r_{n+1}^{2}}\right) W=0
\end{aligned}
$$

Since the number of the independent variables is equal to five: $\left(x, r_{n}^{1}, r_{n}^{2}, r_{n+1}^{1}, r_{n+1}^{2}\right)$, while the number of the equations is three, then system (2.14) possesses two functionally independent solutions $\omega\left(x, n, \bar{r}_{n}, \bar{r}_{n+1}\right)$ and $W\left(x, n, \bar{r}_{n}, \bar{r}_{n+1}\right)$ of first order.

Then we consider the ring $\mathcal{X}$ of dimnension 6 . Without loss of generality we can assume that the base is generated by vector fields (2.13) and a field of form

$$
Y_{4}=\frac{\partial}{\partial r_{n+k}^{1}}+s_{k} \frac{\partial}{\partial r_{n+k}^{2}}+\sum_{l=k+1}^{\infty}\left(s_{l} \frac{\partial}{\partial r_{n+l}^{1}}+d_{l} \frac{\partial}{\partial r_{n+l}^{2}}\right), \quad k \geqslant 1 .
$$

It is clear that the coefficients of the operator $Y_{4}$ are independent of the variables $r_{n, x}^{1}$ and $r_{n, x}^{2}$. Otherwise the dimension of the ring would exceed 6.

In the same way, the coefficients of vector fields $(2.13)$ are independent of $r_{n, x}^{1}$ and $r_{n, x}^{2}$.

If $k \geqslant 2$, we arrive at system (2.14), which possesses two functionally independent solutions of first order.

Let $k=1$. Characteristic equation (2.5) for $x$-integral of first order is reduced to a system consisting of equations (2.14) and equation (see (2.15)

$$
\left(\frac{\partial}{\partial r_{n+1}^{1}}+s_{1} \frac{\partial}{\partial r_{n+1}^{2}}\right) W=0
$$

Since the number of independent variables is equal to five: $\left(x, r_{n}^{1}, r_{n}^{2}, r_{n+1}^{1}, r_{n+1}^{2}\right)$, while the number of equations is equal to four, system (2.14), 2.16) possesses one solution $\omega\left(x, n, \bar{r}_{n}, \bar{r}_{n+1}\right)$ of first order. 
Now we consider equation (2.5) for $x$-integral of second order

$$
\begin{aligned}
& \left(\frac{\partial}{\partial x}+\tilde{\alpha}_{1} \frac{\partial}{\partial r_{n+1}^{1}}+\tilde{\beta}_{1} \frac{\partial}{\partial r_{n+1}^{2}}+\tilde{\alpha}_{2} \frac{\partial}{\partial r_{n+2}^{1}}+\tilde{\beta}_{2} \frac{\partial}{\partial r_{n+2}^{2}}\right) W=0 \\
& \left(\frac{\partial}{\partial r_{n}^{1}}+\gamma_{1} \frac{\partial}{\partial r_{n+1}^{1}}+\delta_{1} \frac{\partial}{\partial r_{n+1}^{2}}+\gamma_{2} \frac{\partial}{\partial r_{n+2}^{1}}+\delta_{2} \frac{\partial}{\partial r_{n+2}^{2}}\right) W=0 \\
& \left(\frac{\partial}{\partial r_{n}^{2}}+p_{1} \frac{\partial}{\partial r_{n+1}^{1}}+q_{1} \frac{\partial}{\partial r_{n+1}^{2}}+p_{2} \frac{\partial}{\partial r_{n+2}^{1}}+q_{2} \frac{\partial}{\partial r_{n+2}^{2}}\right) W=0 \\
& \left(\frac{\partial}{\partial r_{n+1}^{1}}+s_{1} \frac{\partial}{\partial r_{n+1}^{2}}+s_{2} \frac{\partial}{\partial r_{n+2}^{1}}+d_{2} \frac{\partial}{\partial r_{n+2}^{2}}\right) W=0 .
\end{aligned}
$$

Since the number of independent variables is equal to seven: $\left(x, \bar{r}_{n}, \bar{r}_{n+1}, \bar{r}_{n+2}\right)$, and the number of the equations in system (2.17) is equal to four, the latter system possesses three functionally independent solutions $\omega\left(x, n, \bar{r}_{n}, \bar{r}_{n+1}\right), \omega\left(x, n+1, \bar{r}_{n+1}, \bar{r}_{n+2}\right)$ and $W\left(x, n, \bar{r}_{n}, \bar{r}_{n+1}, \bar{r}_{n+2}\right)$. Thus, $x$-integrals $\omega=\omega\left(x, n, \bar{r}_{n}, \bar{r}_{n+1}\right)$ and $W\left(x, n, \bar{r}_{n}, \bar{r}_{n+1}, \bar{r}_{n+2}\right)$ define an entire family of solutions to characteristic equation (2.5).

We proceed to the case $\operatorname{dim} \mathcal{X}=7$. Here the basis of Lie ring $\mathcal{X}$ is generated by the vector fields, which, according to 2.13, 2.15, are of form:

$$
\begin{aligned}
& \tilde{Y}_{1}=\frac{\partial}{\partial x}+\sum_{k=1}^{\infty}\left(\alpha_{k} \frac{\partial}{\partial r_{n+k}^{1}}+\beta_{k} \frac{\partial}{\partial r_{n+k}^{2}}\right) \\
& \tilde{Y}_{2}=\frac{\partial}{\partial r_{n}^{1}}+\sum_{k=1}^{\infty}\left(\gamma_{k} \frac{\partial}{\partial r_{n+k}^{1}}+\delta_{k} \frac{\partial}{\partial r_{n+k}^{2}}\right) \\
& \tilde{Y}_{3}=\frac{\partial}{\partial r_{n}^{2}}+\sum_{k=1}^{\infty}\left(p_{k} \frac{\partial}{\partial r_{n+k}^{1}}+q_{k} \frac{\partial}{\partial r_{n+k}^{2}}\right) \\
& \tilde{Y}_{4}=\frac{\partial}{\partial r_{n+k}^{1}}+s_{k} \frac{\partial}{\partial r_{n+k}^{2}}+\sum_{l=k+1}^{\infty}\left(s_{l} \frac{\partial}{\partial r_{n+l}^{1}}+d_{l} \frac{\partial}{\partial r_{n+l}^{2}}\right) \\
& \tilde{Y}_{5}=\kappa_{l} \frac{\partial}{\partial r_{n+l}^{1}}+\mu_{l} \frac{\partial}{\partial r_{n+l}^{2}}+\sum_{s=n+l+1}^{\infty}\left(\kappa_{s} \frac{\partial}{\partial r_{n+s}^{1}}+\lambda_{s} \frac{\partial}{\partial r_{n+s}^{2}}\right) .
\end{aligned}
$$

Here the coefficients of the vector fields $\tilde{Y}_{i}$ are independent of the variables $r_{n, x}^{1}, r_{n, x}^{2}$ and $l \geqslant k$.

As $k \geqslant 2$, the system of equations

$$
\tilde{Y}_{i} W=0, \quad i=1,2,3,4,5
$$

possesses two functionally independent solutions of first order and any other solution is a function of its shifts, that is,

$$
W=W\left(x, n, \omega^{1}, \omega^{2}, \omega_{1}^{1}, \omega_{1}^{2}, \ldots, \omega_{s}^{1}, \omega_{s}^{2}\right),
$$

where

$$
\begin{aligned}
& \omega^{1}=\omega^{1}\left(x, n, \bar{r}_{n}, \bar{r}_{n+1}\right), \quad \omega^{2}=\omega^{2}\left(x, n, \bar{r}_{n}, \bar{r}_{n+1}\right), \\
& \omega_{i}^{m}=\omega_{i}^{m}\left(x, n+i, \bar{r}_{n+i}, \bar{r}_{n+i+1}\right), \quad m=1,2, \quad i=1,2, \ldots, s .
\end{aligned}
$$

We consider the case $k=1$. If $l \geqslant 3$, then we arrive at system (2.17). Thus, we have an $x$-integral of the first order and an $x$-integral of the second order. They define an entire family of solutions to system (2.18).

Let $l=2$. Then, as above, it is easy to show that sytem (2.18) possesses two solutions $\omega^{1}\left(x, n, \bar{r}_{n}, \bar{r}_{n+1}\right)$ and $\omega^{2}\left(x, n, \bar{r}_{n}, \bar{r}_{n+1}, \bar{r}_{n+2}\right)$, which define all solutions of characteristic equation 
(2.5). It remains to consider the case $l=1$ (as $k=1$ ). If a determinant vanishes:

$$
\left|\begin{array}{ll}
1 & s_{1} \\
\kappa_{1} & \mu_{1}
\end{array}\right|=0
$$

then we replace the operator $\tilde{Y}_{5}$ by $\tilde{\tilde{Y}}_{5}=\tilde{Y}_{5}-\kappa_{1} \tilde{Y}_{4}$. The latter reads as

$$
\tilde{\tilde{Y}}_{5}=\kappa_{l} \frac{\partial}{\partial r_{n+l}^{1}}+\mu_{l} \frac{\partial}{\partial r_{n+l}^{2}}+\sum_{s=n+1+l}^{\infty}\left(\kappa_{s} \frac{\partial}{\partial r_{n+s}^{1}}+\mu_{s} \frac{\partial}{\partial r_{n+s}^{2}}\right)
$$

where $l \geqslant 2$. This case has been studied above.

If

$$
\left|\begin{array}{ll}
1 & s_{1} \\
\kappa_{1} & \mu_{1}
\end{array}\right| \neq 0
$$

then we can replace the operators $\tilde{Y}_{4}$ and $\tilde{Y}_{5}$ by the following ones:

$$
\begin{aligned}
& \tilde{\tilde{Y}}_{4}=\frac{\partial}{\partial r_{n+1}^{1}}+\sum_{l=2}^{\infty}\left(s_{l} \frac{\partial}{\partial r_{n+l}^{1}}+d_{l} \frac{\partial}{\partial r_{n+l}^{2}}\right), \\
& \tilde{\tilde{Y}}_{5}=\frac{\partial}{\partial r_{n+1}^{2}}+\sum_{l=2}^{\infty}\left(\kappa_{l} \frac{\partial}{\partial r_{n+l}^{1}}+\mu_{l} \frac{\partial}{\partial r_{n+l}^{2}}\right) .
\end{aligned}
$$

Thus, we have a system of equations

$$
\tilde{Y}_{1} W=0, \quad \tilde{Y}_{2} W=0, \quad \tilde{Y}_{3} W=0, \quad \tilde{\tilde{Y}}_{4} W=0, \quad \tilde{\tilde{Y}}_{5} W=0 .
$$

For second order solutions $W=W\left(x, n, \bar{r}_{n}, \bar{r}_{n+1}, \bar{r}_{n+2}\right)$ the system contains seven independent variables $x, \bar{r}_{n}, \bar{r}_{n+1}, \bar{r}_{n+2}$, while the number of equations is five. Therefore, there exist two independent integrals of second order.

It is clear that in this case system $(2.19)$ possesses no integrals of the first order. In the same way we consider the case when $\operatorname{dim} \mathcal{X}>7$. Thus, we have proved the following statement.

Lemma 2.2. If the ring $\mathcal{X}$ is finite-dimensional, there exist two independent integrals of minimal order. Any other integral is a function of their shifts.

Lemmata 2.1, 2.2 now imply the following theorem.

Theorem 2.1. System of equations (2.1) possesses two essentially independent $x$-integrals if and only if the ring $\mathcal{X}$ is finite-dimensional.

\section{CharACTERISTIC RINGS}

Let us determine the characteristic ring in the direction $x$ for system (1.1). On the set of locally analytic function functions depending on the variables $x, \bar{r}_{n, x}, \bar{r}_{n}, \bar{r}_{n+1}, \ldots$ the operator of total differentiation in $x$ reads as

$$
D_{x}=\frac{\partial}{\partial x}+\sum_{i=1}^{N} r_{n, x x}^{i} \frac{\partial}{\partial r_{n, x}^{i}}+\sum_{k=0}^{\infty} \sum_{i=1}^{N}\left(r_{n+k, x}^{i} \frac{\partial}{\partial r_{n+k}^{i}}\right) .
$$

System of equations (1.1) yield the relations

$$
\bar{r}_{n+k, x}=\bar{h}_{n+k}\left(x, n, \bar{r}_{n}, \bar{r}_{n+1}, \ldots, \bar{r}_{n+k}, \bar{r}_{n, x}\right), \quad k=1,2, \ldots
$$

We represent operator (3.1) as

$$
D_{x}=\sum_{i=1}^{N} r_{n, x x}^{i} Y_{i}+Y_{N+1}
$$


where

$$
Y_{i}=\frac{\partial}{\partial r_{n, x}^{i}}, \quad Y_{N+1}=\frac{\partial}{\partial x}+\sum_{k=0}^{\infty}\left(r_{n+k, x}^{1} \frac{\partial}{\partial r_{n+k}^{1}}+\cdots+r_{n+k, x}^{N} \frac{\partial}{\partial r_{n+k}^{N}}\right) .
$$

According to formulae (3.2), the vector field $Y_{N+1}$ can be represented as

$$
Y_{N+1}=\frac{\partial}{\partial x}+r_{n, x}^{1} \frac{\partial}{\partial r_{n}^{1}}+\cdots+r_{n, x}^{N} \frac{\partial}{\partial r_{n}^{N}}+\sum_{k=1}^{\infty}\left(\alpha_{k}^{1} \frac{\partial}{\partial r_{n+k}^{1}}+\cdots+\alpha_{k}^{N} \frac{\partial}{\partial r_{n+k}^{N}}\right),
$$

where $\bar{h}_{n+k}=\left(\alpha_{k}^{1}, \ldots, \alpha_{k}^{N}\right)$. We note that

$$
\alpha_{k}^{i}=\alpha_{k}^{i}\left(x, n, \bar{r}_{n}, \bar{r}_{n+1}, \ldots, \bar{r}_{n+k}, \bar{r}_{n, x}\right) .
$$

According (3.3), the characteristic equation

$$
D_{x} W\left(x, n, \bar{r}_{n}, \bar{r}_{n+1}, \ldots, \bar{r}_{n+m}\right)=0
$$

is equivalent to the system

$$
Y_{i} W=0, \quad i=1,2, \ldots, N, N+1 .
$$

With equations (3.5), a Lie ring $\mathcal{X}$ generated by the vectors fields $Y_{i}, i=1,2, \ldots, N, N+1$ is naturally associated. We shall call this ring $\mathcal{X}$ a characteristic Lie $x$-ring of system of equations (1.1). Solutions of equations (3.4) will be called $x$-integrals. The following statement holds.

Lemma 3.1. If system of equations (1.1) possesses $N$ essentially independent $x$-integrals, then the ring $\mathcal{X}$ is finite-dimensional.

Proof. Let system (1.1) admits $N$ essentially independent integrals of the same order $\omega^{i}\left(x, n, \bar{r}_{n}, \ldots, \bar{r}_{n+m}\right)$, that is,

$$
\left|\begin{array}{cccc}
\frac{\partial \omega^{1}}{\partial r_{n+m}^{1}} & \frac{\partial \omega^{1}}{\partial r_{n+m}^{2}} & \cdots & \frac{\partial \omega^{1}}{\partial r_{n+m}^{N}} \\
\cdots & \cdots & \cdots & \cdots \\
\frac{\partial \omega^{N}}{\partial r_{n+m}^{1}} & \frac{\partial \omega^{N}}{\partial r_{n+m}^{2}} & \cdots & \frac{\partial \omega^{N}}{\partial r_{n+m}^{N}}
\end{array}\right| \neq 0 .
$$

Then the identity

$$
\bar{r}_{n+m}=\bar{a}_{n}\left(\omega^{1}, \ldots, \omega^{N}, x, n, \bar{r}_{n}, \ldots, \bar{r}_{n+m-1}\right)
$$

holds. We let $\omega_{n}^{1}=\omega^{1}, \ldots, \omega_{n}^{N}=\omega^{N}$. Then by (3.6) we obtain the relations

$$
\bar{r}_{n+m+k}=\bar{A}_{k}\left(x, n, \omega_{n}^{1}, \ldots, \omega_{n}^{N}, \ldots, \omega_{n+k}^{1}, \ldots, \omega_{n+k}^{N}, \bar{r}_{n}, \bar{r}_{n+1}, \ldots, \bar{r}_{n+m-1}\right),
$$

$k=0,1,2, \ldots$. Thus, taking into consideration formulae (3.7), we pass from independent variables

to new variables

$$
\bar{r}_{n, x}, x, \bar{r}_{n}, \bar{r}_{n+1}, \ldots, \bar{r}_{n+s}, \ldots
$$

$$
\bar{r}_{n, x}, x, \bar{r}_{n}, \bar{r}_{n+1}, \ldots, \bar{r}_{n+m-1}, \omega_{n}^{1}, \ldots, \omega_{n}^{N}, \ldots, \omega_{n+k}^{1}, \ldots, \omega_{n+k}^{N} .
$$

In new variables $(3.9)$, the operator $Y_{N+1}$ is written as

$$
\tilde{Y}_{N+1}=\frac{\partial}{\partial x}+\sum_{k=0}^{m-1}\left(r_{n+k, x}^{1} \frac{\partial}{\partial r_{n+k}^{1}}+\cdots+r_{n+k, x}^{N} \frac{\partial}{\partial r_{n+k}^{N}}\right)
$$

Under the change of variables, the relation holds: $\overline{[X, Z]}=[\bar{X}, \bar{Z}]$, where the bar denotes the initial operator in new variables and the characteristic ring generated by the operators $Y_{1}, Y_{2}, \ldots, Y_{N}, \tilde{Y}_{N+1}$ is finite-dimensional. This is why initial characteristic ring $\mathcal{X}$ is finitedimensional. 
Let initial system of equations (1.1) possesses $N$ essentially independent integrals

$$
\omega^{1}\left(x, n, \bar{r}_{n}, \ldots, \bar{r}_{n+l_{1}}\right), \ldots, \omega^{N}\left(x, n, \bar{r}_{n}, \ldots, \bar{r}_{n+l_{N}}\right)
$$

of different minimal orders $l_{1} \leqslant l_{2} \leqslant \ldots \leqslant l_{N}$. The latter means that the integrals (we denote $\left.l_{N}=M\right)$

$$
\begin{aligned}
& \omega^{1}\left(x, n+M-l_{1}, \bar{r}_{n+M-l_{1}}, \ldots, \bar{r}_{n+M}\right), \\
& \omega^{i}\left(x, n+M-l_{i}, \bar{r}_{n+M-l_{i}}, \ldots, \bar{r}_{n+M}\right), \quad i=1,2, \ldots, N-1, \\
& \omega^{N}\left(x, n, \bar{r}_{n}, \ldots, \bar{r}_{n+M}\right)
\end{aligned}
$$

are essentially independent. Then, passing from variables (3.8) to the variables

$$
\bar{r}_{n, x}, x, \bar{r}_{n}, \bar{r}_{n+1}, \ldots, \bar{r}_{n+m-1}, \omega_{n+m-l_{1}}^{1}, \omega_{n+m-l_{1}+1}^{1}, \ldots, \omega_{n}^{N}, \omega_{n+1}^{N},
$$

as above we obtain that the characteristic ring $\mathcal{X}$ is finite-dimensional. The proof is complete.

Now we consider an inverse problem. The following lemma holds.

Lemma 3.2. If the ring $\mathcal{X}$ is finite-dimensional, then there exist $N$ independent $x$-integrals of minimal order. Any other integral is a function of its shifts.

The scheme of the proof is as follows. Let the ring $\mathcal{X}$ be finite-dimensional. It is clear that $\operatorname{dim} \mathcal{X} \geqslant 2 N+1$. We consider the case $\operatorname{dim} \mathcal{X}=2 N+1$. Then the basis of the $\operatorname{ring} \mathcal{X}$ is given by the vector fields

$$
\begin{gathered}
Y_{1}, Y_{2}, \ldots, Y_{N}, Y_{N+1}, \\
Y_{1, N+1}=\left[Y_{1}, Y_{N+1}\right], Y_{2, N+1}=\left[Y_{2}, Y_{N+1}\right], \ldots, Y_{N, N+1}=\left[Y_{N}, Y_{N+1}\right] .
\end{gathered}
$$

Since

$$
\begin{aligned}
Y_{N+1}= & \frac{\partial}{\partial x}+\sum_{i=1}^{N} r_{n, x}^{i} \frac{\partial}{\partial r_{n}^{i}}+\sum_{k=1}^{\infty}\left(\alpha_{k}^{1}\left(x, n, \bar{r}_{n}, \bar{r}_{n+1}, \ldots, \bar{r}_{n+k}, \bar{r}_{n, x}\right) \frac{\partial}{\partial r_{n+k}^{1}}+\cdots\right. \\
& \left.\cdots+\alpha_{k}^{N}\left(x, n, \bar{r}_{n}, \bar{r}_{n+1}, \ldots, \bar{r}_{n+k}, \bar{r}_{n, x}\right) \frac{\partial}{\partial r_{n+k}^{N}}\right)
\end{aligned}
$$

then

$$
\begin{aligned}
& {\left[Y_{1}, Y_{N+1}\right]=\frac{\partial}{\partial r_{n}^{1}}+\sum_{k=1}^{\infty}\left(\frac{\partial \alpha_{k}^{1}}{\partial r_{n, x}^{1}} \frac{\partial}{\partial r_{n+k}^{1}}+\cdots+\frac{\partial \alpha_{k}^{N}}{\partial r_{n, x}^{1}} \frac{\partial}{\partial r_{n+k}^{N}}\right),} \\
& {\left[Y_{i}, Y_{N+1}\right]=\frac{\partial}{\partial r_{n}^{i}}+\sum_{k=1}^{\infty}\left(\frac{\partial \alpha_{k}^{1}}{\partial r_{n, x}^{i}} \frac{\partial}{\partial r_{n+k}^{1}}+\cdots+\frac{\partial \alpha_{k}^{N}}{\partial r_{n, x}^{i}} \frac{\partial}{\partial r_{n+k}^{N}}\right), \quad i=1,2, \ldots, N}
\end{aligned}
$$

We replace the vector field $Y_{N+1}$ by

$$
\tilde{Y}_{N+1}=Y_{N+1}-r_{n, x}^{1} Y_{1, N+1}-r_{n, x}^{2} Y_{2, N+1}-\cdots-r_{n, x}^{N} Y_{N, N+1}
$$


Thus, we have the following basis:

$$
\begin{aligned}
& Y_{1}=\frac{\partial}{\partial r_{n, x}^{1}}, \quad Y_{2}=\frac{\partial}{\partial r_{n, x}^{2}}, \quad \ldots, \quad Y_{N}=\frac{\partial}{\partial r_{n, x}^{N}} \\
& \tilde{Y}_{N+1}=\frac{\partial}{\partial x}+\sum_{k=1}^{\infty}\left(\tilde{\alpha}_{k}^{1} \frac{\partial}{\partial r_{n+k}^{1}}+\cdots+\tilde{\alpha}_{n+k}^{N}\right) \\
& Y_{1, N+1}=\frac{\partial}{\partial r_{n}^{1}}+\sum_{k=1}^{\infty}\left(p_{1, k}^{1} \frac{\partial}{\partial r_{n+k}^{1}}+\cdots+p_{1, k}^{N} \frac{\partial}{\partial r_{n+k}^{N}}\right) \\
& Y_{2, N+1}=\frac{\partial}{\partial r_{n}^{2}}+\sum_{k=1}^{\infty}\left(p_{2, k}^{1} \frac{\partial}{\partial r_{n+k}^{1}}+\cdots+p_{2, k}^{N} \frac{\partial}{\partial r_{n+k}^{N}}\right) \\
& \ldots \quad \\
& Y_{N, N+1}=\frac{\partial}{\partial r_{n}^{N}}+\sum_{k=1}^{\infty}\left(p_{N, k}^{1} \frac{\partial}{\partial r_{n+k}^{1}}+\cdots+p_{N, k}^{N} \frac{\partial}{\partial r_{n+k}^{N}}\right)
\end{aligned}
$$

It is easy to see that the coefficients $p_{j, k}^{i}, i=1,2, \ldots, N, j=1,2, \ldots, N$ are independent of the variables $x, n, \bar{r}_{n}, \ldots, \bar{r}_{n+k}$, otherwise $\operatorname{dim} \mathcal{X}>2 N+1$.

According (3.11), characteristic equation (3.4) for $x$-integral $W\left(x, n, \bar{r}_{n}, \bar{r}_{n+1}\right)$ is reduced to the system of equations

$$
\begin{aligned}
& \left(\frac{\partial}{\partial x}+\tilde{\alpha}_{1}^{1} \frac{\partial}{\partial r_{n+1}^{1}}+\cdots+\tilde{\alpha}_{1}^{N} \frac{\partial}{\partial r_{n+1}^{N}}\right) W=0 \\
& \left(\frac{\partial}{\partial r_{n}^{1}}+p_{1,1}^{1} \frac{\partial}{\partial r_{n+1}^{1}}+\cdots+p_{1,1}^{N} \frac{\partial}{\partial r_{n+1}^{N}}\right) W=0 \\
& \quad \cdots \\
& \left(\frac{\partial}{\partial r_{n}^{N}}+p_{N, 1}^{1} \frac{\partial}{\partial r_{n+1}^{1}}+\cdots+p_{N, 1}^{N} \frac{\partial}{\partial r_{n+1}^{N}}\right) W=0
\end{aligned}
$$

Since the number of independent variables is equal to $2 N+1: x, r_{n}^{1}, \ldots, r_{n}^{N}, r_{n+1}^{1}, \ldots, r_{n+1}^{N}$, and the number of equations is equal to $N+1$, then system (3.12) possesses $N$ functionally independent solutions

$$
\omega^{1}\left(x, n, \bar{r}_{n}, \bar{r}_{n+1}\right), \ldots, \omega^{N}\left(x, n, \bar{r}_{n}, \bar{r}_{n+1}\right)
$$

of first order.

Similar to the proof Lemma 2.2 one can consider the cases $\operatorname{dim} \mathcal{X}>2 N+1$.

Thus, Lemmata 3.1, 3.2 imply the following statement.

Theorem 3.1. System of equations (1.1) possesses $N$ essentially independent $x$-integrals if and onyl if the ring $\mathcal{X}$ is finite-dimensional.

\section{Conclusion}

Nowadays, the techniques based on characteristic Lie rings are an effective tool for studying the integrability of nonlinear models, both continuous (equations and systems) [1], [2], [4], [7], [8], and semi-discrete equations [5], 6]. The criterion of integrability of semi-discrete systems considered in the present work seems to be as follows: system of equations (1.1) possesses a complete set of integrals ( $N$ integrals in each characteristic direction) if and only if the characteristic ring in each direction is finite-dimensional. In the work we have proved the first part of this criterion concerning a continuous characteristic direction. 


\section{BIBLIOGRAPHY}

1. A.N. Leznov, V.G. Smirnov, A.B. Shabat. The group of internal symmetries and the conditions of integrability of two-dimensional dynamical systems // Teor. Matem. Fiz. 51:1, 10-21 (1982). [Theor. Math. Phys. 51:1, 322-330 (1982).]

2. A.B. Shabat, R.I. Yamilov. Exponential systems of type I and Cartan matrices // Preprint. Bashkir Branch of Academy of Sciences of USSR. (1981). (in Russian).

3. I. Habibullin, N. Zheltukhina, A. Sakieva. Discretization of hyperbolic type Darboux integrable equations preserving integrability // J. Math. Phys. 52:9, 093507 (2011).

4. A.V. Zhiber, O.S. Kostrigina. Exactly solvable models of wave processes // Vestnik UGATU. 7:25, 83-89 (2007). (in Russian).

5. I. Habibullin, N. Zheltukhina, A. Pekcan. On the classification of Darboux integrable chains // J. Math. Phys. 49:10, 102702 (2008).

6. I. Habibullin, N. Zheltukhina, A. Pekcan. Complete list of Darboux integrable chains of the form $t_{1, x}=t_{x}+d\left(t, t_{1}\right) / /$ J. Math. Phys. 50:10, 102710 (2009).

7. A.V. Zhiber, A.M. Gurieva. On characteristic equation of a quasi-linear hyperbolic system of equations // Vestnik UGATU. 6:2, 26-34 (2005). (in Russian).

8. A.V. Zhiber, O.S. Kostrigina. Characteristic algebras of nonlinear hyperbolic systems of equations // Zhurn. SFU. Ser. Matem. Fiz. 3:2, 173-184 (2010).

9. I.T. Habibullin, A.R. Khakimova. Characteristic Lie algebras of integrable differential-difference equations in 3D // J. Phys. A: Math. Theor. 54:29, 295202 (2021).

Anatoly Vasilievich Zhiber,

Institute of Mathematics,

Ufa Federal Research Center, RAS,

Chernyshevky str. 112,

450008, Ufa, Russia

E-mail: zhiber@mail.ru

Maria Nikolaevna Kuznetsova,

Institute of Mathematics,

Ufa Federal Research Center, RAS,

Chernyshevky str. 112,

450008, Ufa, Russia

E-mail: mariya.n.kuznetsova@gmail.com 